J. Clin. Chem. Clin. Biochem.

Vol. 16, 1978, pp. 551-555

\title{
Vergleichsuntersuchung zur Thyrotropin-Bestimmung an 6 kommerziell erhältlichen Testpackungen
}

\author{
Von E. Leicht und G. Biro
}

Medizinische Klinik und Poliklinik - Innere Medizin II - der Universität des Saarlandes, 6650 Homburg-Saar

(Eingegangen am 23. März/10. Juli 1978)

Zusammenfassung: An 6 kommerziell erhältlichen Testpackungen zur Thyrotropin-Bestimmung wurde eine Vergleichsuntersuchung durchgeführt. Qualität der Standards und Reproduzierbarkeit der Standardkurven waren befriedigend; dagegen bestanden erhebliche Unterschiede in der für die Empfindlichkeit der Methoden entscheidenden Steilheit der Standardkurven im klinisch wichtigen Bereich um und unter $10 \mathrm{mE} / 1$. Bei Testpackungen mit flacherer Standardkurve waren Intra-assay-Variation und Wiederfinderate schlechter. Die übereinstimmend in fast allen Testpackungen angegebene Intra-assay-Variation von 3-8\% im normalen Bereich wurde bei keinem der untersuchten Kits auch nur annähernd erreicht.

Deutliche methodische Verbesserungen dürften bei entsprechenden Modifikationen (optimales Verhältnis der Tracerund Antikörper-Konzentrationen, Vorinkubation) und bei Berücksichtigung eines bisher immer noch unerfültten grundsätzlichen Erfordernisses (Standardverdünnung in Thyrotropin-freiem menschlichen Plasma) verhältnismäßig leicht zu erreichen sein.

\section{A comparison of six commercially available thyrotropin radioimmunoassay kits}

Summary: A comparative investigation of 6 thyrotropin-kits, commercially available in the Federal Republic Germany, was carried out. Quality of thyrotropin standards and reproducibility of calibration curves were satisfactory. Considerable differences were found in the slope of the calibration curves and, in consequence, in the sensitivity of the methods in the clinically important range around and below $10 \mathrm{mU} / 1$. With kits having flat calibration curves, intraassay-variation was high and recovery inconsistent. An intraassay-variation of 3-8\%, as advertized by almost all manufactures, was not nearly achieved with any of the methods.

Improvements may be achieved with appropriate modifications (use of optimal concentrations of tracer and antibody, and preincubation) and by taking into account a basic requirement that has hitherto been ignored, i. e. the dilution of standards in human plasma free of thyrotropin.

\section{Einleitung}

Die radioimmunologische Bestimmung von Thyrotropin vor und nach Verabreichung von Thyroliberin hat sich einen festen Platz in der Funktionsdiagnostik der Schilddrüse erworben (1-5). Voraussetzung ist eine empfindliche und reproduzierbare Bestimmungsmethode. In letzter Zeit wurden zahlreiche vorgefertigte Testpackungen zuir Thyrotropin-Bestimmung kommerziell angeboten; es erschien uns daher notwendig, eine Vergleichsuntersuchung durchzuführen, um eine Übersicht über Empfindlichkeit, Reproduzierbarkeit, technischen Aufwand und klinische Brauchbarkeit der verschiedenen Methoden zu gewinnen.

\section{Material}

Testpackungen zur. Thyrotropin-Bestimmung der Firmen Abbott Lab., Behringwerke AG, BIO-RAD, Corning, Diagnostic Products, Schwarz-Mann.
Kontroll-Seren: NMS (Nuclear Medical Systems) II a RIA Control, lot Nr. RolA (angegebene Konzentration 10-14 mU/1). Lederle Diagnostics RIA Control Serum III. lot Nr. 2947-300 (angegebene Konzentration 2-2,5 mU/l). Thyrotropin-Standard MRC 68/38. Relefact-TRHR, Hoechst AG, Frankfurt/Main.

\section{Methoden}

Für jede Testpackung wurden Standardkurven unter strengster Einhaltung der Gebrauchsanleitung angefertigt. Um einen Vergleich zu ermöglichen, wurde, auch wenn von der Herstellerfirma anders angegeben, für jeden Standardpunkt das Verhältnis von Antikörper-gebundener Radioaktivität zur maximalen Tracerbindung $\left(B \times \mathrm{B}_{0}^{-1}\right)$ bestimmt. Die Standardkurven wurden durch kübische Spline-Glättung approximiert. Zur Bestimmung der Präzision in Serie wurden mit jeder Testpackung an beiden Kontrollseren in einem Ansatz 20-fach-Bestimmungen durchgeführt; Mittelwerte $(\overline{\overline{\mathbf{X}}})$, Standardabweichungen (s), mittlerer Fehler des Mittelwertes $\left(s_{\bar{x}}\right)$ und Variationskoeffizienten $\left(V K=s \cdot \bar{x}^{-1} \cdot 100\right)$ wurden berechnet. Die Reproduzierbarkeit der Standardkurven wurde für jede Testpackung 4 Tage später geprüft. Für Wiederauffindungsuntersuchungen wurde das Kontrollserum mit niedriger Thyrotropin-Konzentration um 2 bzw. $5 \mathrm{mE} / 1 \mathrm{mit}$ MRCStandard 68/38 aufgestockt; die Konzentrationen vor und nach 
Aufstockung wurden mit jeder Testpackung im gleichen Ansatz gemessen. Die angegebenen Werte (Tab. 3) sind aus 4 Bestimmungen gemittelt. Die prozentuale Wiederauffindungsrate $(W)$ wurde nach der Formel $\mathrm{W}(\%)=(\mathrm{G}-\mathrm{V}) \cdot \mathrm{z}^{-1} \cdot 100$ berechnet. Dabei bedeutet $\mathrm{V}$ die gefundene Thyrotropin-Konzentration in $\mathrm{mE} / \mathrm{l}$ vor, $G$ jene nach Aufstockung und $Z$ die Thyrotropin-Konzentration in $\mathrm{mE} / \mathrm{l}$, um die das Kontrollserum aufgestockt wurde. Die Bestimmung von Trijodthyronin und Thyroxin erfolgte radioimmunologisch mit Testpackungen der Firma Corning. Der Normalbereich für Trijodthyronin betrug 1,2-3,4 $\mathrm{nmol} / 1$, jener für Thyroxin $64,5-155 \mathrm{nmol} / 1$.

\section{Ergebnisse und Diskussion}

Die Werte für $\mathrm{B} \mathrm{x} \mathrm{B}_{\mathrm{o}}{ }^{-1}$ der mit den 6 verschiedenen Testpackungen erstellten Standardkurven sind Tabelle $1 \mathrm{zu}$ entnehmen. Die für die Empfindlichkeit der Methode maßgebende Steilheit der Kurven zeigt im klinisch wichtigen Bereich um und unter $10 \mathrm{mE} / \mathrm{l}$ erhebliche Unterschiede. Der Variationskoeffizient im Bereich um $2 \mathrm{mE} / 1$ ist bei Testpackungen mit wenig empfindlicher Standardkurve sehr hoch (Tab. 2). Im Bereich um $12 \mathrm{mE} / 1$ sind die Variationskoeffizienten für alle Testpackungen deutlich niedriger, doch bestehen auch hier erhebliche Unterschiede (Tab. 2). Unverständlicherweise wird in fast allen Arbeitsanleitungen ein Intrassay-Variationskoeffizient von 3-8\% im Bereich normaler Werte angegeben. Ein so gutes Ergebnis konnte mit keiner der untersuchten Testpackungen auch nur annähernd erzielt werden. Die mit den verschiedenen Methoden gefundenen Mittelwerte für die Konzentrationen der Kontrollseren stimmten gut überein (Tab. 2).
Dies spricht für gute Übereinstimmung der beigegebenen Standards. Die Wiederfindung ist bei Kits mit flacher Standardkurve deutlich schlechter (Tab. 3). Die Reproduzierbarkeit der Standardpunkte war bei allen untersuchten Testpackungen befriedigend (Tab. 1).

Tabelle 4 zeigt die Thyrotropin-Werte im Serum bei 16 Patienten vor und 30 Minuten nach Verabreichung von $400 \mathrm{mg}$ Thyroliberin. Anhand der basalen und stimulierten Thyrotropin-Werte konnten bei den meisten $\mathrm{Pa}$ tienten übereinstimmende diagnostische Folgerungen gezogen werden. So waren die Thyrotropin-Konzentrationen bei 2 Patienten mit Hypothyreose aufgrund aller Methoden erhöht und vermehrt stimulierbar (Pat. 1 und 3, Tab. 4). Bei einem Patienten mit kompensiertem Schilddrüsenadenom (Pat. 8) wurde übereinstimmend normale, bei 4 Patienten mit ausgeprägter Hyperthyreose (Pat. 2, 5, 10, 16), 2 Patienten mit dekompensiertem autonomen Adenom (Pat. 13, 14) sowie bei 2 Patienten unter Schilddrüsenhormonsubstitution (Pat. 11, 12) fehlende Stimulierbarkeit gefunden. Bei 2 Patienten mit Rezidivstruma fiel die Thyrotropin-Antwort nach Thyroliberin-Gabe negativ aus, obwohl szintigraphisch kein autonomes Adenom nachweisbar war (Pat. 4,7). Offenbar bestehen bei diesen Patienten teilweise autonom-sezernierende Schilddrüsenanteile (6).

Bei 3 Untersuchten (Pat. 6, 9, 15) wurde die Frage der Stimulierbarkeit bei Zugrundelegung eines Mindestwertes von $\Delta$ Thyroliberin $>2 \mathrm{mE} / 1$ durch die verschiedenen Testpackungen unterschiedlich beantwortet. Wurde dagegen ein Thyrotropin-Anstieg um mindestens $4 \mathrm{mE} / 1$ ge-

Tab. 1. Stark unterschiedlicher Bindungsabfall $\left(\mathrm{B} \mathrm{x} \mathrm{Bo}^{-1}\right)$ im klinisch wichtigen Bereich bis und um $10 \mathrm{mE} / 1$. Gute Reproduzierbarkeit der Standardkurven bei erneutem Ansatz 4 Tage später (jeweils 2. Spalte).

\begin{tabular}{|c|c|c|c|c|c|c|c|c|c|c|c|c|}
\hline \multirow[b]{2}{*}{$\mathrm{mE} / \mathrm{l}$} & \multicolumn{2}{|c|}{ Abbott-Lab. } & \multicolumn{2}{|c|}{ Behringwerke AG } & \multicolumn{2}{|c|}{ BIO-Rad } & \multicolumn{2}{|c|}{ Corning } & \multicolumn{2}{|c|}{ Diagnostic Products } & \multicolumn{2}{|c|}{ Schwarz-Mann } \\
\hline & $\mathrm{B} / \mathrm{B}_{\mathrm{O}}(\mathrm{I})$ & $\mathrm{B} / \mathrm{B}_{\mathrm{O}}$ (II) & $\mathrm{B} / \mathrm{B}_{\mathrm{O}}(\mathrm{I})$ & $\mathrm{B} / \mathrm{B}_{\mathrm{o}}$ (II) & $\mathrm{B} / \mathrm{B}_{\mathrm{O}}(\mathrm{I})$ & $\mathrm{B} / \mathrm{B}_{\mathrm{o}}$ (II) & $\mathrm{B} / \mathrm{B}_{\mathrm{o}}(\mathrm{I})$ & $\mathrm{B} / \mathrm{B}_{\mathrm{o}}$ (II) & $\mathrm{B} / \mathrm{B}_{\mathrm{O}}$ (I) & $\mathrm{B} / \mathrm{B}_{\mathrm{o}}$ (II) & $\mathrm{B} / \mathrm{B}_{\mathrm{o}}(\mathrm{I})$ & $\mathrm{B} / \mathrm{B}_{\mathrm{o}}$ (II) \\
\hline 0 & 1,00 & 1,00 & 1,00 & 1,00 & 1,00 & 1,00 & 1,00 & 1,00 & 1,00 & 1,00 & 1,00 & 1,00 \\
\hline 1,5 & - & - & - & - & - & - & 0,83 & 0,80 & - & - & 0,85 & 0,83 \\
\hline 1,65 & - & - & 0,93 & 0,95 & - & - & - & - & - & - & - & - \\
\hline 2,0 & - & - & - & - & 0,93 & 0,93 & - & - & 0,95 & 0,94 & - & - \\
\hline 2,5 & 0,85 & 0,87 & - & - & - & - & - & - & - & - & - & - \\
\hline 3,0 & - & - & - & - & - & - & 0,72 & 0,71 & - & - & - & - \\
\hline 3,3 & - & - & 0,94 & 0,95 & - & - & - & - & - & - & - & - \\
\hline 4,0 & - & - & - & - & - & - & - & - & - & - & 0,63 & 0,64 \\
\hline 5,0 & 0,74 & 0,78 & - & - & 0,86 & 0,86 & - & - & 0.86 & 0,85 & - & - \\
\hline 6,0 & - & - & - & - & - & - & 0,57 & 0,56 & $=$ & - & - & - \\
\hline 6,6 & - & - & 0,84 & 0,82 & - & - & - & - & - & - & - & - \\
\hline 10,0 & 0,62 & 0,59 & - & - & 0,77 & 0,77 & - & - & 0,75 & 0,75 & 0,44 & 0,43 \\
\hline 13,2 & - & - & 0,72 & 0,71 & - & - & - & - & - & - & - & - \\
\hline 15,0 & - & - & - & - & - & - & 0,40 & 0,40 & - & - & - & - \\
\hline 20,0 & 0,53 & 0,51 & - & - & $-\cdots$ & - & - & - & 0,61 & 0,61 & 0,32 & 0,32 \\
\hline 25,0 & - & - & - & - & 0,51 & 0,52 & - & - & - & - & - & - \\
\hline 26,4 & - & - & 0,52 & 0,51 & - & - & - & - & - & - & - & - \\
\hline 30,0 & - & - & - & - & - & - & 0,31 & 0,31 & - & - & - & - \\
\hline 40,0 & 0,43 & 0,45 & - & - & - & - & - & - & 0,45 & 0,44 & - & - \\
\hline 50,0 & - & - & - & - & 0,32 & 0,31 & - & - & - & - & 0,27 & 0,27 \\
\hline 52,8 & - & - & 0,40 & 0,37 & - & - & - & - & - & - & $\therefore$ & - \\
\hline 60,0 & - & $\tau$ & - & - & - & - & 0,27 & 0,26 & - & - & - & - \\
\hline 100,0 & - & $\dot{-}$ & - & - & - & - & - & - & 0,33 & 0,31 & - & - \\
\hline 105,6 & - & - & 0,28 & 0,27 & - & - & - & - & - & - & - & - \\
\hline
\end{tabular}


Tab. 2. Mittelwerte, Standardabweichungen und Variationskoeffizienten bei 20-fach-Bestimmung der Thyrotropin-Konzentrationen zweier Kontrollseren mit den 6 verschiedenen Testpackungen. Bei wenig empfindlicher Standardkurve (Tab. 1) sind die Variationskoeffizienten sehr hoch. Die Mittelwerte $(\overline{\mathbf{x}})$ zeigen verhältnismäßig gute Übereinstimmung.

\section{Intraassay I}

Lederle RIA Control Serum III Lot Nr. 2947-300

Angegebene Konzentration 2,0-2,5 mE/1

\begin{tabular}{|c|c|c|c|c|c|c|}
\hline & Abbott Lab. & Behringwerke AG & BIO-RAD & Corning & Diagnostic Products & Schwarz/Mann \\
\hline $\begin{array}{l}\mathrm{n} \\
\overline{\mathrm{x}}(\mathrm{mE} / \mathrm{l}) \\
\mathrm{s}(\mathrm{mE} / \mathrm{l}) \\
\mathrm{s}_{\overline{\mathrm{x}}}(\mathrm{mE} / \mathrm{l}) \\
\mathrm{VK}(\%)\end{array}$ & $\begin{array}{r}20 \\
2,49 \\
0,42 \\
0,09 \\
16,82\end{array}$ & $\begin{array}{r}20 \\
2,46 \\
1,23 \\
0,28 \\
50,10\end{array}$ & $\begin{array}{l}20 \\
2,12 \\
0,89 \\
0,20 \\
42,12\end{array}$ & $\begin{array}{l}20 \\
2,02 \\
0,34 \\
0,07 \\
16,77\end{array}$ & $\begin{array}{r}20 \\
2,43 \\
0,98 \\
0,22 \\
40,33\end{array}$ & $\begin{array}{r}20 \\
1,95 \\
0,32 \\
0,07 \\
16,16\end{array}$ \\
\hline
\end{tabular}

Intraassay II

Kontrollserum(*NMS IIaRIA Control, Lot Nr. Ro1A)

Angegebene Konzentration 10,0-14,0 mE/1

\begin{tabular}{lllllll} 
& Abbott Lab. & Behringwerke AG & BIO-RAD & Corning & Diagnostic Products & Schwarz/Mann \\
\hline $\mathbf{n}$ & 20 & 20 & 20 & 20 & 20 & 20 \\
$\overline{\mathbf{x}}(\mathrm{mE} / 1)$ & 11,97 & 11,86 & 12,91 & 12,01 & 11,88 & 12,07 \\
$\mathrm{~s}(\mathrm{mE} / 1)$ & 1,42 & 2,26 & 0,75 & 0,94 & 2,00 & 1,20 \\
$\mathrm{~s}_{\overline{\mathbf{x}}(\mathrm{mE} / 1)}$ & 0,32 & 0,51 & 0,17 & 0,21 & 0,45 & 0,27 \\
$\mathrm{VK}(\%)$ & 11,87 & 19,06 & 5,80 & 7,85 & 16,79 & 9,90 \\
\hline
\end{tabular}

*NMS = Nuclear Medical Systems, Inc. USA

Tab. 3. Ergebnisse der Wiederauffind ungsversuche.

\begin{tabular}{llllll} 
& Z & S & G & G-V & W \\
\hline \multirow{3}{*}{ Abbott } & 0,0 & 2,49 & - & - & - \\
& 2,0 & 4,49 & 3,78 & 1,29 & 64,5 \\
& 5,0 & 7,49 & 8,04 & 5,55 & 111,0 \\
Behringwerke AG & 0,0 & 2,46 & - & - & - \\
& 2,0 & 4,46 & 3.22 & 0,76 & 38,0 \\
& 5,0 & 7,46 & 5,31 & 2,85 & 57,0 \\
BIO-RAD & 0,0 & 2,12 & - & - & - \\
& 2,0 & 4,12 & 5,07 & 2,95 & 147,5 \\
& 5,0 & 7,12 & 7,96 & 5,84 & 116,8 \\
Corning & 0,0 & 2,02 & - & - & - \\
& 2,0 & 4,02 & 3,71 & 1,69 & 84,5 \\
& 5,0 & 7,02 & 7,58 & 5,56 & 111,2 \\
Diagnostic Products & 0,0 & 2,43 & - & - & - \\
& 2,0 & 4,43 & 5,92 & 3,49 & 174,5 \\
& 5,0 & 7,43 & 6,14 & 3,71 & 74,2 \\
Schivarz/Mann & 0,0 & 1,95 & - & - & - \\
& 2,0 & 3,95 & 4,27 & 2,32 & 116,0 \\
& 5,0 & 6,95 & $\mathbf{8 , 1 0}$ & $\mathbf{6 , 1 5}$ & 123,0 \\
\hline & & & & &
\end{tabular}

wobei: $Z$ = Zugegebene Thyrotropin-Menge in $\mathrm{mE} / 1$

$\mathbf{S}=$ Sollwert (Kontrolserum $+Z$ in $\mathrm{mE} / \mathrm{l}$ )

$\mathbf{G}=$ Radioimmunologisch gefundene ThyrotropinKonzentration in $\mathrm{mE} / \mathrm{l}$

$\mathbf{V}=\mathbf{X}=$ Vorhandene Thyrotropin-Konzentration in $\mathrm{mE} / 1$ vor Zugabe Z

$W=$ Wiederauffindungsrate in $\%=[(G-V) / Z] \cdot 100$ fordert, waren die Thyrotropin-Konzentrationen dieser Patienten übereinstimmend nicht stimulierbar. Die unterschiedliche Beurteilung ist verständlich, da durch die verschiedenen Methoden statt $2 \mathrm{mE} / 1$ 0,76-3,49 mE/l, statt $5 \mathrm{mE} / 1$ 2,85-6,15 mE/1 wiedergefunden wurden (Tab. 3).

Diese Ergebnisse beleuchten die Schwierigkeit, den zu fordernden Konzentrationsunterschied für die Annahme eines sogenannten positiven Thyroliberin-Testes genau anzugeben. Gefordert wird von verschiedenen Autoren ein Anstieg um mindestens $2-4 \mathrm{mE} / 1(7,8,6)$ bzw. um etwa 50\% des Ausgangswertes (9) aus dem erniedrigten bzw. niedrig-normalen Bereich. Würde in vorliegendem Falle der Mindestanstieg mit $4 \mathrm{mE} / \mathrm{l}$ festgesetzt, wäre ein falsch positiver Thyroliberin-Test bei allen Methoden selten. Bei Annahme von etwa 1,5 mE/1 dürfte dagegen ein falsch negatives Ergebnis selten auftreten. Offensichtlich sind die Testpackungen der Firmen Corning, SchwarzMann und Abbott in der Lage, einen Mindestanstieg um $2-3 \mathrm{mE} / 1(7,8)$ zu erfassen; für die übrigen 3 Testpackungen erscheint dies zumindest zweifelhaft. Für die vollständige Trennung der Kollektive ist offenbar keine der untersuchten Methoden empfindlich genug; sie ist wahrscheinlich aus Gründen der biologischen Variation auch nicht möglich. Auch mit dem zur Zeit empfindlichsten Verfahren für die Thyrotropin-Bestimmung, dem zytochemischen Bioassay, wurden widersprüchliche Befunde berichtet (10). 
Tab. 4. Thyrotropin-Konzentrationen vor (I) und $30 \mathrm{~min}$ nach (II) intravenöser Verabreichung von $0,4 \mathrm{mg}$ Thyroliberin bei $16 \mathrm{~Pa}$ tienten mit verschiedenen Funktionszuständen der Schilddrüse, bestimmt mit den 6 Testpackungen. Bei Patient 5 wurde Thyrotropin vor, sowie $10,20,30 \mathrm{~min}$ nach Thyroliberin-Gabe bestimmt.

\begin{tabular}{|c|c|c|c|c|c|c|c|c|c|c|}
\hline Pat. & Diagnose & $\begin{array}{l}\text { Triiod- } \\
\text { thyronin } \\
(\mathrm{nmol} / \mathrm{l})\end{array}$ & $\begin{array}{l}\text { Thyroxi } \\
\text { (nmol/l) }\end{array}$ & & Abbott & $\begin{array}{l}\text { Behring- } \\
\text { werke AG }\end{array}$ & $\begin{array}{l}\text { yrotropin (m } \\
\text { BIO-RAD }\end{array}$ & E/l) & $\begin{array}{l}\text { Diagnostic } \\
\text { Products }\end{array}$ & $\begin{array}{l}\text { Schwarz- } \\
\text { Mann }\end{array}$ \\
\hline 1 & Hypothyreose & 2,13 & 87,5 & II & $\begin{array}{l}11,6 \\
>40\end{array}$ & $\begin{array}{l}12,0 \\
86,4\end{array}$ & $\begin{aligned} & 15,7 \\
> & 50\end{aligned}$ & $\begin{array}{l}16,9 \\
>60\end{array}$ & $\begin{array}{l}13,1 \\
97,2\end{array}$ & $\begin{aligned} & 14,3 \\
> & 50\end{aligned}$ \\
\hline 2 & Hyperthyreose & $>12,3$ & 388,3 & II & $\begin{array}{l}3,7 \\
3,3\end{array}$ & $\begin{array}{l}2,1 \\
3,2\end{array}$ & $\begin{array}{l}2,4 \\
3,0\end{array}$ & $\begin{array}{l}2,8 \\
3,2\end{array}$ & $\begin{array}{l}1,6 \\
2,8\end{array}$ & $\begin{array}{l}2,2 \\
3,0\end{array}$ \\
\hline 3 & Hypothyreose & 2,31 & 37,4 & II & $\begin{aligned} & 38,1 \\
> & 40\end{aligned}$ & $\begin{array}{r}44,7 \\
>105,6\end{array}$ & $\begin{aligned} & 28,7 \\
> & 50\end{aligned}$ & $\begin{aligned} & 40,4 \\
> & 60\end{aligned}$ & $\begin{aligned} & 34,0 \\
> & 100\end{aligned}$ & $\begin{aligned} & 32,9 \\
> & 50\end{aligned}$ \\
\hline 4 & Rezidivstruma & 2,31 & 110,9 & II & $\begin{array}{l}1,5 \\
1,1\end{array}$ & $\begin{array}{l}2,2 \\
2,9\end{array}$ & $\begin{array}{l}2,1 \\
1,3\end{array}$ & $\begin{array}{l}1,3 \\
1,1\end{array}$ & $\begin{array}{l}1,5 \\
1,0\end{array}$ & $\begin{array}{l}0,8 \\
0,9\end{array}$ \\
\hline 5 & Hyperthyreose & 7,5 & 232,2 & $\underset{\text { III }}{\text { II }}$ & $\begin{array}{l}3,48 \\
1,79 \\
2,27 \\
3,15\end{array}$ & $\begin{array}{l}3,37 \\
4,70 \\
3,13 \\
3,42\end{array}$ & $\begin{array}{l}2,11 \\
2,80 \\
2,37 \\
1,48\end{array}$ & $\begin{array}{l}2,47 \\
2,59 \\
2,20 \\
2,43\end{array}$ & $\begin{array}{l}2,94 \\
3,71 \\
2,64 \\
2,07\end{array}$ & $\begin{array}{l}3,29 \\
2,74 \\
2,25 \\
2,29\end{array}$ \\
\hline 6 & Strumektomie 1967 & 1,62 & 139,2 & II & $\begin{array}{l}3,1 \\
6,0\end{array}$ & $\begin{array}{l}3,4 \\
3,9\end{array}$ & $\begin{array}{l}4,0 \\
5,5\end{array}$ & $\begin{array}{l}3,4 \\
5,0\end{array}$ & $\begin{array}{l}2,2 \\
4,1\end{array}$ & $\begin{array}{l}2,3 \\
6,2\end{array}$ \\
\hline 7 & Rezidivstruma & 2,50 & 131,6 & II & $\begin{array}{l}3,2 \\
3,9\end{array}$ & $\begin{array}{l}2,4 \\
3,0\end{array}$ & $\begin{array}{l}2,9 \\
3,3\end{array}$ & $\begin{array}{l}1,0 \\
2,4\end{array}$ & $\begin{array}{l}2,2 \\
3,0\end{array}$ & $\begin{array}{l}1,7 \\
2,3\end{array}$ \\
\hline 8 & $\begin{array}{l}\text { Komp. autonomes } \\
\text { Adenom }\end{array}$ & 2,53 & 113,5 & II & $\begin{array}{r}2,9 \\
13,8\end{array}$ & $\begin{array}{r}3,3 \\
13,5\end{array}$ & $\begin{array}{r}2,2 \\
14,0\end{array}$ & $\begin{array}{r}1,2 \\
12,0\end{array}$ & $\begin{array}{r}2,4 \\
13,3\end{array}$ & $\begin{array}{r}3,3 \\
10,0\end{array}$ \\
\hline 9 & Strumektomie 1960 & 3,54 & 180,6 & $\begin{array}{l}\text { I } \\
\text { II }\end{array}$ & $\begin{array}{l}\phi \\
2,6\end{array}$ & $\begin{array}{l}\phi \\
1,0\end{array}$ & $\begin{array}{l}0,5 \\
2,0\end{array}$ & $\begin{array}{l}\phi \\
1,8\end{array}$ & $\begin{array}{l}1,4 \\
3,7\end{array}$ & $\begin{array}{l}\phi \\
2,2\end{array}$ \\
\hline 10 & Hyperthyreose & 10,9 & 276,1 & $\underset{\text { II }}{\text { I }}$ & $\begin{array}{l}3,3 \\
3,7\end{array}$ & $\begin{array}{l}2,4 \\
2.9\end{array}$ & $\begin{array}{l}3,4 \\
3,8\end{array}$ & $\begin{array}{l}1,6 \\
1,9\end{array}$ & $\begin{array}{l}2,0 \\
2,7\end{array}$ & $\begin{array}{l}2,9 \\
2,7\end{array}$ \\
\hline 11 & Strumektomie 1965 & 4,0 & 144,5 & I & $\begin{array}{l}2,7 \\
2,1\end{array}$ & $\begin{array}{l}4,0 \\
4,7\end{array}$ & $\begin{array}{l}2,2 \\
2,8\end{array}$ & $\begin{array}{l}4,2 \\
4,0\end{array}$ & $\begin{array}{l}3,1 \\
3,9\end{array}$ & $\begin{array}{l}3,7 \\
3,7\end{array}$ \\
\hline 12 & Hypothyreose & 2,93 & 200,0 & II & $\begin{array}{l}1,1 \\
2,0\end{array}$ & $\begin{array}{l}3,7 \\
4,9\end{array}$ & $\begin{array}{l}3,0 \\
3,6\end{array}$ & $\begin{array}{l}2,7 \\
2,9\end{array}$ & $\begin{array}{l}3,3 \\
3,1\end{array}$ & $\begin{array}{l}3,0 \\
3,1\end{array}$ \\
\hline 13 & $\begin{array}{l}\text { dekomp. autonomes } \\
\text { Adenom }\end{array}$ & 3,10 & 158,7 & II & $\begin{array}{l}2,4 \\
3,7\end{array}$ & $\begin{array}{l}3,7 \\
2,2\end{array}$ & $\begin{array}{l}3,0 \\
3,9\end{array}$ & $\begin{array}{l}1,1 \\
1,1\end{array}$ & $\begin{array}{l}3,0 \\
3,3\end{array}$ & $\begin{array}{l}1,9 \\
2,1\end{array}$ \\
\hline 14 & $\begin{array}{l}\text { dekomp. autonomes } \\
\text { Adenom }\end{array}$ & 3,54 & 121,3 & II & $\begin{array}{l}2,0 \\
2,5\end{array}$ & $\begin{array}{l}3,7 \\
5,1\end{array}$ & $\begin{array}{l}2,4 \\
2,1\end{array}$ & $\begin{array}{l}1,4 \\
1,7\end{array}$ & $\begin{array}{l}1,8 \\
1,8\end{array}$ & $\begin{array}{l}0,5 \\
1,2\end{array}$ \\
\hline 15 & $\begin{array}{l}\text { szintigr. komp. } \\
\text { autonomes Adenom }\end{array}$ & 2,29 & 109,7 & I & $\begin{array}{l}2,1 \\
4,9\end{array}$ & $\begin{array}{l}5,6 \\
6,8\end{array}$ & $\begin{array}{l}3,7 \\
4,7\end{array}$ & $\begin{array}{l}1,9 \\
3,0\end{array}$ & $\begin{array}{l}2,9 \\
6,1\end{array}$ & $\begin{array}{l}1,4 \\
4,8\end{array}$ \\
\hline 16 & Hyperthyreose & 12,3 & 316,1 & II & $\begin{array}{l}2,7 \\
2,2\end{array}$ & $\begin{array}{l}3,9 \\
3,0\end{array}$ & $\begin{array}{l}2,2 \\
2,8\end{array}$ & $\begin{array}{l}1,2 \\
1,1\end{array}$ & $\begin{array}{l}3,4 \\
3,3\end{array}$ & $\begin{array}{l}0,8 \\
1,0\end{array}$ \\
\hline
\end{tabular}

Tab. 5. Übersicht über technische Besonderheiten der untersuchten Testpackungen.

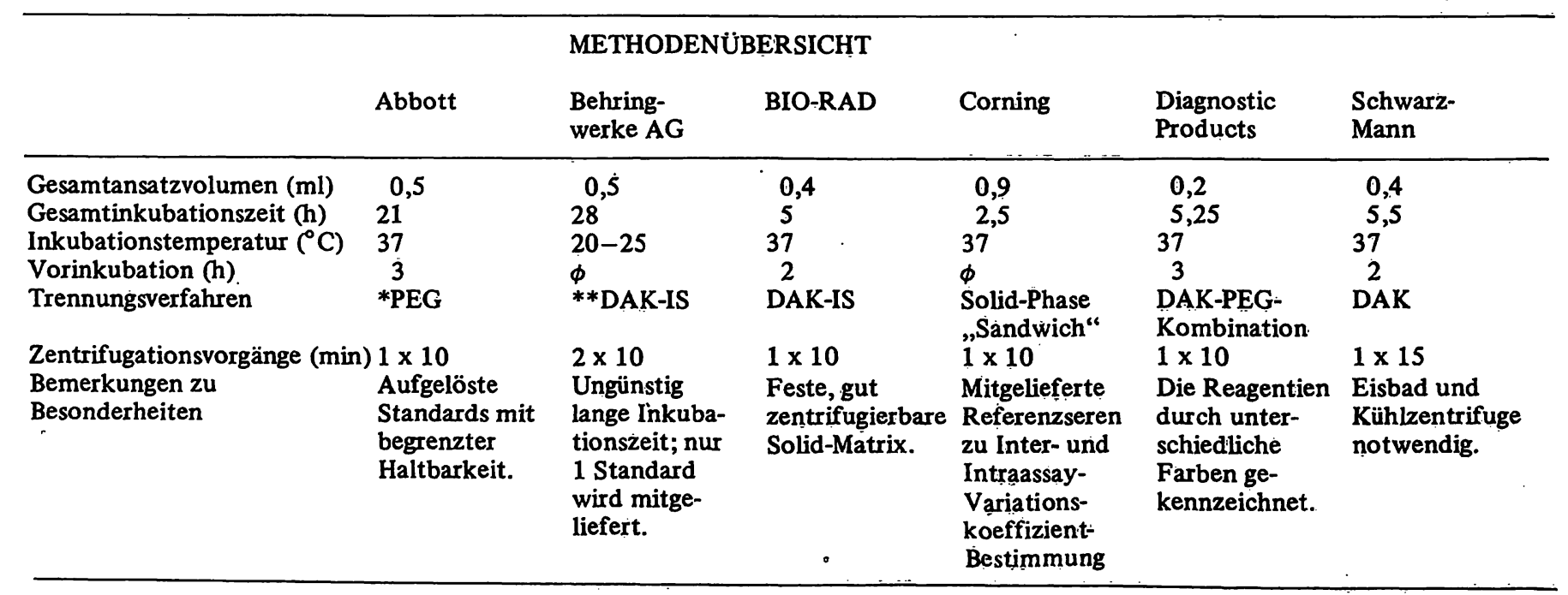

* PEG = Polyethylenglykol Fällung

DAK $=$ Doppelantikörper

** DAK-IS = Doppelantikörper Immunosorbent 
Einige für die praktische Anwendung wichtige technische Daten zeigt Tabelle 5 . Unterschiede betreffen besonders den Zeitaufwand sowie Zahl und Art der Darbietung der Standards. Zum Teil ist die Aufarbeitungszeit schlecht mit dem normalen 8-Stunden-Tag zu vereinbaren. Am schnellsten durchzuführen ist der Corning "Sandwich". Assay. Bei dem Kit von Diagnostic Products sind die Reagenzien durch unterschiedliche Farben gekennzeichnet, was einen Pipettierfehler sofort erkennen läßt.

Die vorliegende Vergleichsuntersuchung wurde durch die offensichtlich unterschiedliche Qualität kommerzieller Testpackungen zur Thyrotropin-Bestimmung angeregt. $\mathrm{Da}$ die Untersuchung aus praktischen Gründen auf 6 Testpackungen beschränkt werden mußte, wird kein Anspruch auf eine vollständige Ubersicht über das derzeitige Angebot in der Bundesrepublik Deutschland erhoben. Die jetzt verfügbaren Methoden stellen gegenüber dem Stand des Jahres 1975 (11) einen deutlichen Fortschritt dar. Dennoch muß die gegenwärtige Lage als unbefriedigend angesehen werden, da mehrere im Handel befindliche Testpackungen nicht allen von der Klinik zu stellenden Anforderungen genügen.

\section{Literatur}

1. Anderson, M. S., Bowers, C. Y., Kastin, A. J., Schalch, D. S., Schally, A. V., Snyder, P. J., Utiger, R. D., Wilber, J. F. \& Wise, A. J. (1971), N. Engl. J. Med. 285, 1279-1283.

2. Burger, H. G. \& Patel, Y.C. (1977), Clinics in endocrinology and metabolism 6, 83-100.

3. Hesch, R.-D., Emrich, D., von zur Mühlen A. \& Breuel, H.-P. (1975), Dtsch. Med. Wochenschr. 100, 805-812.

4. Rothenbuchner, G., Birk, J., Loos, U., Raptis, S. \& Pfeiffer, E. F. (1976), Therapiewoche $26,2105-2123$.

5. Smyth, P. P. A. \& O'Donovan, D. K. (1973), J. Irish Med. Ass. 66, 670-674.

6. Joseph, K., Mahlstedt, J., Pries, H.-H. \& Schmidt, U. (1976), Compact news in nuclear medicine 7, 71-77.
Der wesentliche Grund dafür ist die Unempfindlichkeit der Methoden im klinisch wichtigen Bereich. Dafür sind vor allem drei wichtig erscheinende Ursachen anzufuhren:

1. Immer noch werden vorgefertigte Standards in unterschiedlichen proteinhaltigen Lösungen (Schweine-, Pferdeplasma, albuminhaltiger Puffer) dargeboten. Dies führt zum Abweichen der Verdünnungskurven für Standards gegenüber jenen für Thyrotropin-haltiges menschliches Serum, besonders im unteren Normbereich. Die im menschlichen Serum gefundenen Konzentrationen werden dadurch etwas angehoben. Es ist unumgänglich, die Standards in Thyrotropin-freiem menschlichem Plasma zu verdünnen (12).

2. Fehlende oder zu kurzfristige Vorinkubation ohne markiertes Hormon. Durch diesen Schritt wäre wahrscheinlich eine höhere Empfindlichkeit erreichbar.

3. Bei günstiger Einstellung der Konzentrationen von markiertem Antigen und / oder des Antikörpers ist bei einigen Testpackungen eine Verbesserung der Meßempfindlichkeit sicher zu erwarten.

7. Hartmann, K. P., Henderkott, U., Hör, G. \& Bottermann, P. (1975), Verh. Dtsch. Ges. Inn. Med. 81, 1559-1563.

8. Hüfner, M., Munzinger, H., Papke, H., Barwich, D., Bahner, F., Schenk, P. \& Röher, D. (1975), Radiologe 15, 245-250. 9. Emrich, D. (1976), Med. Welt 27, 1716-1719.

10. Von zur Mühlen, A., Hashimoto, T., Emrich, E. \& Döhler, K.-D. (1976), Acta Endocrinol. (Copenhagen) 108, 110-111.

11. Marschner, I., Erhardt, F. W. \& Scriba, P. C. (1976), J. Clin. Chem. Clin. Biochem. 14, 345-351.

12. Erhardt, F. W. \& Scriba, P. C. (1974), Ärztl. Lab. 20, 191198.

Dr. E. Leicht

Med. Univ.-Klinik und Poliklinik, $6650 \mathrm{Homburg} / \mathrm{Saar}$ 
- 